\title{
Rosmarinic acid ameliorates hyperglycemia and insulin sensitivity in diabetic rats, potentially by modulating the expression of PEPCK and GLUT4
}

This article was published in the following Dove Press journal:

Drug Design, Development and Therapy

7 July 2016

Number of times this article has been viewed

\author{
Joshua Runtuwene ${ }^{1,2}$ \\ Kai-Chun Cheng' \\ Akihiro Asakawa' \\ Haruka Amitani' \\ Marie Amitani' \\ Akinori Morinaga' \\ Yoshiyuki Takimoto ${ }^{3}$ \\ Bernabas Harold Ralph \\ Kairupan ${ }^{2}$ \\ Akio Inui' \\ 'Department of Psychosomatic \\ Internal Medicine, Kagoshima \\ University Graduate School of \\ Medical and Dental Sciences, \\ Kagoshima, Japan; ${ }^{2}$ Faculty of Medicine, \\ Sam Ratulangi University, Manado, \\ Indonesia; ${ }^{3}$ Department of Biomedical \\ Ethics, Graduate School of Medicine, \\ The University of Tokyo, Tokyo, Japan
}

Correspondence: Akio Inui Department of Psychosomatic Internal Medicine, Kagoshima University Graduate School of Medical and Dental Sciences, 8-35-I Sakuragaoka, Kagoshima 890-8520, Japan

Tel +8I 99275575 I

Fax +8I 992755749

Email inui@m.kufm.kagoshima-u.ac.jp
Background: Rosmarinic acid (RA) is a natural substance that may be useful for treating diabetes mellitus. The present study investigated the effects of RA on glucose homeostasis and insulin regulation in rats with streptozocin (STZ)-induced type 1 diabetes or high-fat diet (HFD)-induced type 2 diabetes.

Methods: Glucose homeostasis was determined using oral glucose tolerance tests and postprandial glucose tests, and insulin activity was evaluated using insulin tolerance tests and the homeostatic model assessment for insulin resistance. Additionally, the protein expression levels of PEPCK and GLUT4 were determined using Western blot analysis.

Results: RA administration exerted a marked hypoglycemic effect on STZ-induced diabetic rats and enhanced glucose utilization and insulin sensitivity in HFD-fed diabetic rats. These effects of RA were dose-dependent. Meanwhile, RA administration reversed the STZ- and HFDinduced increase in PEPCK expression in the liver and the STZ- and HFD-induced decrease in GLUT4 expression in skeletal muscle.

Conclusion: RA reduces hyperglycemia and ameliorates insulin sensitivity by decreasing PEPCK expression and increasing GLUT4 expression.

Keywords: diabetes mellitus, STZ, HFD, HOMA-IR, PEPCK, GLUT4

\section{Introduction}

Diabetes mellitus (DM) is one of the most common metabolic disorders. Its prevalence has grown as a result of the increased consumption of high-calorie foods and the increased adoption of a sedentary lifestyle. Pharmacological approaches to this disease frequently induce drug-related problems that are primarily seen in patients receiving multiple drugs to control their medical conditions. Complementary or alternative treatments, such as herbal compounds, are frequently used to treat diabetes. ${ }^{1}$ In fact, one of the most commonly used drugs, metformin, is plant derived. Therefore, herbal medicine may be a promising source of compounds that are more effective and less harmful than existing drugs and that may constitute innovative remedies for treating DM.

Rosmarinic acid (RA) is a potent antioxidant that is present in many common culinary herbs, such as those that belong to Lamiaceae, Boraginaceae, and Anthocerotaceae families. RA was first isolated from rosemary (Rosmarinus officinalis), a widely used culinary plant. $^{2}$ Recently, studies examining the utility of RA to treat diabetic conditions have suggested that RA may reduce diabetes-induced disorders and complications. In diabetic animals, initial studies showed that RA may control plasma glucose by modulating SGLT1 trafficking to the intestinal brush-border membrane ${ }^{3}$ 
to ameliorate hyperglycemia (HG). ${ }^{4}$ Moreover, RA has been shown to protect endothelial functions ${ }^{5}$ and to prevent diabetes-induced sexual disorders by elevating serum testosterone levels, ${ }^{6}$ primarily via its antioxidative effects. Furthermore, RA protects against diabetic glomerular deterioration ${ }^{7}$ and nephropathy, ${ }^{7,8}$ and it confers neuroprotective effects under ischemic conditions ${ }^{9}$ and in diabetic neuropathy. ${ }^{10}$ Additionally, it mitigates oxidative diabetes-induced brain damage. ${ }^{11}$ RA also protects pancreatic cells from glucolipotoxicity. ${ }^{12}$ These findings indicate that RA may ameliorate DM-induced disorders and complications. However, the mechanism(s) potentially underlying the RA-induced amelioration of DM remains unclear.

To explore the role of RA in diabetes treatments, the present study investigated the effect of RA on glucose homeostasis and insulin regulation in two animal models of diabetes: streptozocin (STZ)-induced type 1-like diabetes in rats and high-fat diet (HFD)-induced type 2-like diabetes in rats. We performed oral glucose tolerance tests (OGTTs) and postprandial glucose tests (PGTs) to evaluate glucose utilization, and we used insulin tolerance tests (ITTs) to evaluate insulin sensitivity and the homeostatic model assessment (HOMA) to characterize insulin resistance. Additionally, the protein expression levels of PEPCK and GLUT4 were determined using Western blot analysis to investigate the potential mechanism(s) underlying the effects of RA on glucose homeostasis.

\section{Materials and methods}

\section{Animals}

Seven-week-old male Wistar rats weighing 170-210 g were obtained from Japan SLC, Inc. (Hamamatsu, Japan). These rats were housed individually in a pathogen-free, temperature- $\left(25^{\circ} \mathrm{C}\right)$ and humidity-controlled room on a 12-hour light/12-hour dark cycle (lights on at $7 \mathrm{am}$ ) at the animal center of Kagoshima University (Kagoshima, Japan). The animals had free access to food and tap water. All experimental procedures were approved by the Ethics Committee for Animal Care and Use of Kagoshima University (institutional review board approval number MD15017) and conducted in accordance with the Japanese National Standardized Guidelines for Animal Experiments of Kagoshima University.

\section{Induction of diabetes}

After animals had fasted (48 hours), type 1-like diabetes was induced using a single intraperitoneal injection of STZ (65 mg/kg; Sigma-Aldrich, St Louis, MO, USA) that was freshly dissolved in a citrate buffer $(10 \mathrm{mM}, \mathrm{pH} 4.5)$. On the seventh day after STZ administration, blood glucose concentrations were evaluated in samples obtained from tails using a blood glucose meter. Only rats with a basal blood glucose level $>300 \mathrm{mg} / \mathrm{dL}$ were considered diabetic.

An insulin-resistant model was induced by feeding the rats with a HFD (60\% kcal fat; D12492; Research Diets Inc., New Brunswick, NJ, USA) ad libitum for 8 weeks. The altered animals were then validated: HFD rats showed significant gains in body weight, accumulated body fat, and impaired glucose tolerance (fasting glucose $\geq 130 \mathrm{mg} / \mathrm{dL}$ ).

\section{Experimental procedure and drug administration}

OnceHGwasachieved(DM1 $\geq 300 \mathrm{mg} / \mathrm{dL}, \mathrm{HFD} \geq 130 \mathrm{mg} / \mathrm{dL}$ ), the animals were assigned to a working group $(n=8)$, and each group was treated with RA at the indicated dose for 7 days. RA ( $\geq 98 \%$ purified by high-performance liquid chromatography; Sigma-Aldrich) was dissolved in saline and administrated via intraperitoneal injection once a day for 7 days. The control group was administered saline. Additionally, to investigate the chronic effects of RA, additional STZ and HFD groups that displayed HG were treated with RA at the indicated dose for 28 days. Then, changes in body weight and variations in daily food and water intake were evaluated on the 28th day and compared to the values that were obtained on the first day prior to treatment with RA.

Once treatment was complete, the animals were fasted for 8 hours and then anesthetized using an intraperitoneal injection of pentobarbital $(150 \mathrm{mg} / \mathrm{kg})$. After blood samples were obtained via heart puncture, the animals were sacrificed using cervical dislocation. Serum was collected in heparinized tubes containing heparin and maintained on ice. The samples were centrifuged at $3,000 \times g$ for 15 minutes at $4{ }^{\circ} \mathrm{C}$, and then the top yellow plasma layer was carefully pipetted and placed in a new tube. Liver samples were also collected from the right ventral lobe, and skeletal muscle samples were taken from soleus muscle. All samples were frozen in isopentane, cooled in liquid nitrogen, and stored at $-80^{\circ} \mathrm{C}$ until further processing. To investigate its chronic effects, RA was administered to rats for 28 days after a confirmation of diabetes, and food and water intake was measured during the RA administration period. No infusion-related side effects were observed during RA treatment.

\section{Measurements of biochemical parameters}

To study the effectiveness of RA at different doses in modulating hypoglycemic activity, postprandial blood glucose (PGT) was estimated daily during the RA treatment period in the STZ rats. 
Fasting animals ( 8 hours) were fed a bolus of $180 \mathrm{mg} / \mathrm{dL}$ of glucose, and 2 hours later, their blood was drained and tested to determine the concentration of glucose.

To understand the acute effects of RA on glucose modulation, OGTTs were performed in the normal and STZ groups. The rats were fasted for 8 hours, and D-glucose $(2 \mathrm{~g} / \mathrm{kg})$ was then administered via intragastric gavage to each rat. Blood samples were drawn from the tail vein of each rat at 0,30 , 60,120 , and 180 minutes after the administration of glucose, and glucose levels were measured. The OGTT was performed after 7 days of treatment with RA. The area-under-the-curve (AUC) value of glucose was determined using the total AUC from the sampling period from 0 to 120 minutes.

Plasma glucose levels were measured using a blood glucose meter (NIPRO, Osaka, Japan), and insulin levels were determined using enzyme-linked immunosorbent assay (ELISA) with a rat insulin ELISA kit (Mercodia AB, Uppsala, Sweden) according to the manufacturer's instructions. To determine whether RA treatment improved insulin resistance, we assessed the results of the ITT and the HOMA for insulin resistance (HOMA-IR) on the first and last days of RA treatment. In the ITT experiment, after the rats were fasted for 8 hours, they were intraperitoneally injected with insulin at $0.5 \mathrm{IU} / \mathrm{kg}$ body weight, and blood samples were collected at $0,15,30$, and 60 minutes to measure glucose levels. The HOMA index was calculated as the fasting glucose concentration $(\mathrm{mmol} / \mathrm{L}) \times$ the fasting insulin concentration $(\mathrm{U} / \mathrm{mL}) / 22.5$, with the insulin resistance of normal subjects assumed to be 1 , as previously described. ${ }^{13}$

\section{Western blot analysis}

The expression of PEPCK and GLUT4 was characterized using Western blot analysis as previously described. ${ }^{14}$ Frozen muscle samples $(100 \mathrm{mg})$ were homogenized in $1 \mathrm{~mL}$ of lysis buffer. The homogenate was centrifuged at $1,000 \times \mathrm{g}$ for 5 minutes to pellet the nuclei and insoluble material. The supernatant was moved to a new tube and further centrifuged at $100,000 \times g$ for 1 hour to pellet the membranes. The supernatant from this step was collected as the cytosolic fraction that was used in the assay. Protein concentrations were measured using bicinchoninic acid protein assay methods (Thermo Fisher Scientific, Waltham, MA, USA). Protein samples were filtered and transferred to membranes via sodium dodecyl sulfate-polyacrylamide gel electrophoresis (10\% acrylamide gels) in a Bio-Rad Trans-Blot system (Bio-Rad Laboratories, Inc., Tokyo, Japan). The membranes were submerged in 5\% nonfat milk in Tris-buffered saline containing $0.1 \%$ Tween 20 (TBS-T) for $\sim 1$ hour. They were then washed in TBS-T and hybridized with primary antibodies (diluted in Tris-buffered saline) for 16 hours. The following specific antibodies were used to characterize protein expression: PEPCK (anti-rabbit, 1:1,000 dilution; Santa Cruz Biotechnology Inc., Dallas, TX, USA) and GLUT4 (anti-mouse, 1:1,000 dilution; Abcam plc, Cambridge, UK). Additionally, the membranes were incubated with an antibody for $\beta$-actin (Sigma-Aldrich) (1:5,000 dilution) as the internal control. Incubation with secondary antibodies and antigen-antibody complex detection were performed using an electrochemiluminescence kit (Thermo Fisher Scientific). The immunoblots for PEPCK (62 kDa), GLUT4 (30 kDa), and $\beta$-actin (42 kDa) were compared to those for anti- $\beta$-actin (1:1,000; Santa Cruz Biotechnology), which was used as an internal control.

\section{Statistical analysis}

Statistical analyses were performed using SPSS software (SPSS Inc., Chicago, IL, USA). Between-group differences were analyzed using one-way analysis of variance followed by Dunnett or Tukey-Kramer multiple comparisons tests. A post hoc least significant difference test was used to analyze insulin and HOMA-IR. OGTT responses were analyzed using repeated measures analysis of variance. The results are reported as the mean \pm standard error of the mean and were considered significant at $P<0.05$.

\section{Results}

\section{RA improved glucose homeostasis in the diabetic groups}

Figure 1 demonstrates that PGT was performed via an intraperitoneal injection of a $20 \%$ glucose solution

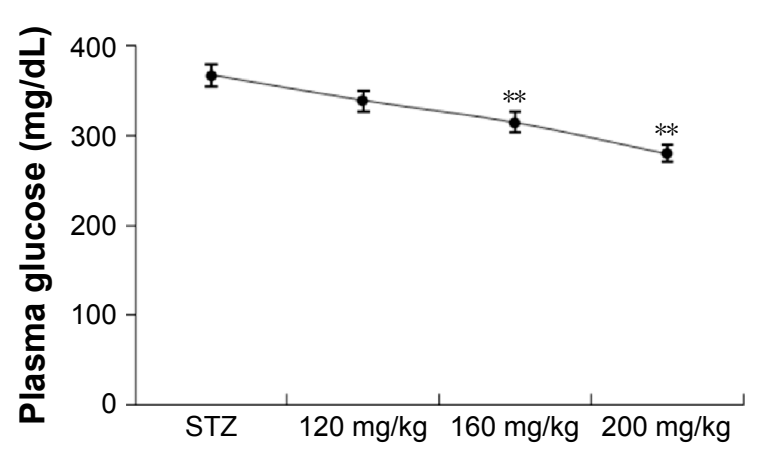

Figure I Dose-response effects of RA on plasma glucose level in STZ-rats. Notes: STZ rats were administered saline or RA (120, 160, or $200 \mathrm{mg} / \mathrm{kg}$ ) via an intraperitoneal injection. Plasma glucose levels were determined at $I 20$ minutes after RA treatment. The data are expressed as the means $\pm \operatorname{SEM}(n=8) . * * P<0.0$ I, significantly different from the value in the respective saline-administered STZ rats (control).

Abbreviations: RA, rosmarinic acid; STZ, streptozocin; SEM, standard error of the mean. 
A

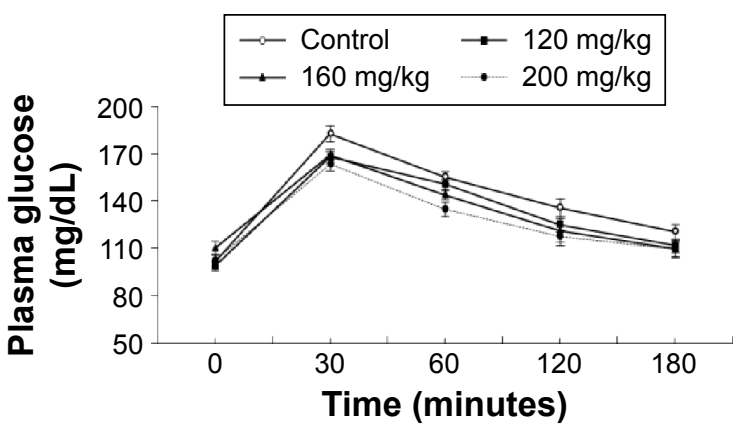

C

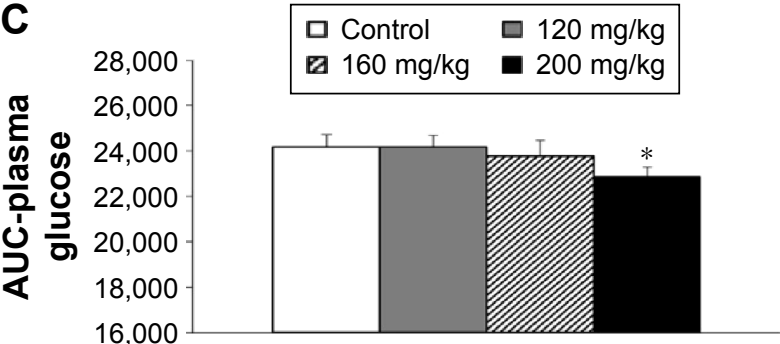

B
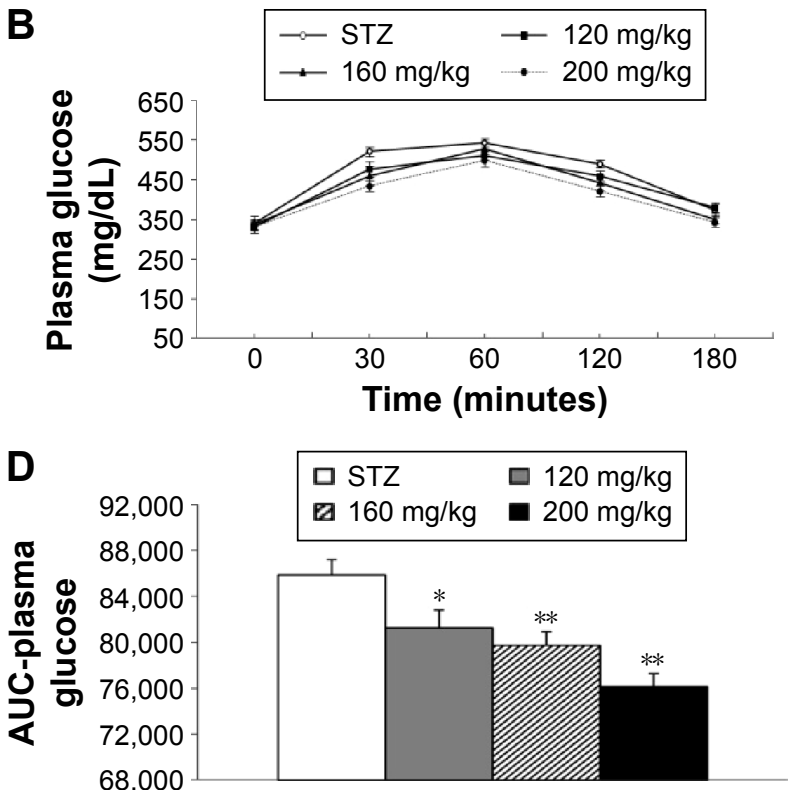

Figure 2 Effect of RA on the OGTT in normal and STZ rats.

Notes: After an 8-hour fast, normal and STZ-induced diabetic mice were intragastrically administered with equal volumes of $0.9 \%$ saline or different doses of RA (I20, I60, and $200 \mathrm{mg} / \mathrm{kg}$ ). After 15 minutes, D-glucose $(2 \mathrm{~g} / \mathrm{kg})$ was orally administered for the OGTT. The results represent the OGTT in (A) normal and (B) diabetic mice, and (C and D) the corresponding calculated relative AUC for glucose concentration. The results are expressed as the means $\pm S E M$ ( $n=8$ ); $* P<0.05$ and $* * P<0.0 \mathrm{I}$ compared to the control group.

Abbreviations: RA, rosmarinic acid; OGTT, oral glucose tolerance test; STZ, streptozocin; AUC, area under the curve; SEM, standard error of the mean.

( $2 \mathrm{mg} / \mathrm{g}$ of body weight) at 1 week after STZ administration. Plasma glucose levels decreased and reached a minimum value at 120 minutes. The higher dose of RA $(200 \mathrm{mg} / \mathrm{kg})$ resulted in a lower glucose level than the $160 \mathrm{mg} / \mathrm{kg}$ dose. It therefore appears that $200 \mathrm{mg} / \mathrm{kg}$ of RA is the most effective dose for affecting postprandial blood glucose in STZ rats. Thus, we found that RA had a pronounced dose-dependent hypoglycemic effect.

In pharmacology studies, OGTT was performed after the STZ rats and normal rats were treated with RA (Figure 2) to demonstrate the therapeutic efficiency of RA. The OGTT showed that the maximal blood glucose levels at 30 minutes after glucose administration were $183 \pm 5.12 \mathrm{mg} / \mathrm{dL}$ in normal rats and $522 \pm 11.99 \mathrm{mg} / \mathrm{dL}$ in diabetic rats. Pretreatment with RA improved glucose tolerance in a dose-dependent manner (Figure 2A and B) and significantly reduced the calculated relative area under the glucose concentration curve (AUC) (Figure $2 \mathrm{C}$ and $\mathrm{D}$ ) in both normal and diabetic rats.

In the HFD group, ITT was conducted once a hyperglycemic condition was confirmed to determine the effect of RA on insulin sensitivity. The blood glucose level in the RA-administered group (160 and $200 \mathrm{mg} / \mathrm{kg}$ ) was significantly different and remained lower after insulin loading than the level in the diabetic rats at the 15- and 30-minute time points (Figure 3). These results indicate that RA enhanced insulin sensitivity in diabetic rats. The effect of RA on insulin sensitivity was greater at higher doses.

\section{RA reversed insulin resistance in the insulin-resistant group}

Plasma glucose and insulin levels were measured to determine the effect of RA on insulin sensitivity in HFD-fed rats. Before the administration of RA, the rats in all groups were

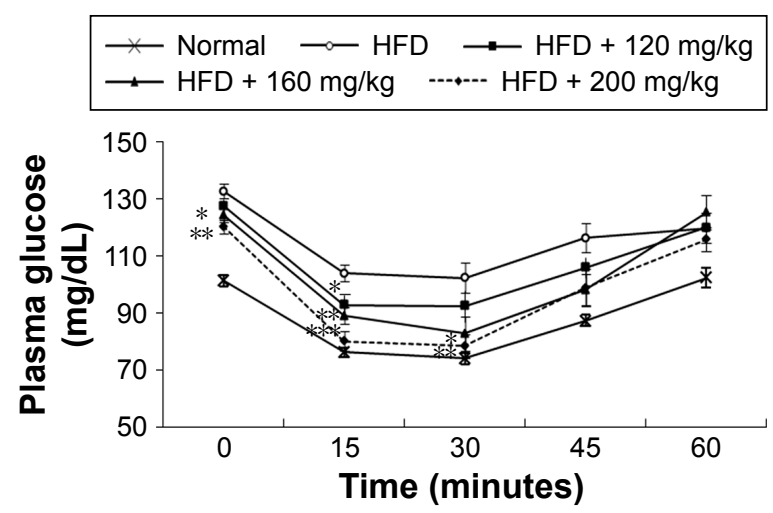

Figure 3 Insulin tolerance test in HFD-fed diabetic rats after 7 days of RA treatment at the indicated doses.

Notes: The values indicate the mean \pm SEM $(n=8) . * P<0.05, * * p<0.01$, and *** $P<0.005$ compared to the saline-administered HFD rat control group, which received the same volume of vehicle.

Abbreviations: HFD, high-fat diet; RA, rosmarinic acid; SEM, standard error of the mean. 
Table I Effect of rosmarinic acid on blood glucose, plasma insulin, and HOMA-IR levels in the experimental groups of rats after a 7-day experimental period

\begin{tabular}{lllll}
\hline Groups & Normal & HFD & HFD + & HFD + \\
& & & $\mathbf{1 2 0} \mathbf{~ m g / k g}$ & $\mathbf{2 0 0} \mathbf{~ m g / k g}$ \\
\hline Glucose $(\mathrm{mmol} / \mathrm{L})$ & $5.71 \pm 0.16$ & $7.42 \pm 0.13$ & $7.04 \pm 0.10$ & $6.67 \pm 0.08$ \\
Insulin $(\mu \mathrm{U} / \mathrm{mL})$ & $36.60 \pm 1.10$ & $74.76 \pm 2.83$ & $66.65 \pm 4.11$ & $59.01 \pm 3.8 \mathrm{I}$ \\
HOMA-IR & $9.12 \pm 0.24$ & $24.70 \pm 1.14$ & $20.76 \pm 1.09$ & $17.50 \pm 1.18$ \\
\hline
\end{tabular}

Note: The values are shown as the mean $\pm \operatorname{SEM}(n=8)$.

Abbreviations: HOMA-IR, homeostatic model assessment for insulin resistance; HFD, high-fat diet; SEM, standard error of the mean.

confirmed to be markedly hyperglycemic, with fasting plasma glucose levels $>130 \mathrm{mg} / \mathrm{dL}$. The insulin and plasma glucose levels after 7 days of RA treatment were significantly lower than the levels in the control group (insulin $537.76 \pm 20.71$ vs $409.79 \pm 26.49 \mathrm{pmol} / \mathrm{L}$ and fasting plasma glucose $133 \pm 3.61$ vs $120 \pm 2.41 \mathrm{mg} / \mathrm{dL}$, both shown before RA treatment vs after 7 days of RA treatment; $P<0.05$ ). ITT was conducted once a hyperglycemic condition was confirmed to determine the effect of RA on insulin sensitivity. Compared to the chow diet group, the HFD-fed rats were insulin resistant. Within the HFD group, the blood glucose level in the RA-administered group (160 and $200 \mathrm{mg} / \mathrm{kg}$ ) was significantly different after insulin loading and remained lower than the level in the diabetic rats at the 15- and 30-minute time points (Figure 3). The mean values of the analyzed parameters (insulin, glycemia, HOMA-IR) in the study groups are shown in Table 1. These data indicate that RA enhanced insulin sensitivity in diabetic rats. The effect of RA on insulin sensitivity was greater at higher doses.

In addition, a comparison of the effects of lowest and highest dosage ( 120 and $200 \mathrm{mg} / \mathrm{kg}$ ) of RA revealed a dose-related effect on plasma insulin and glucose levels (data not shown).

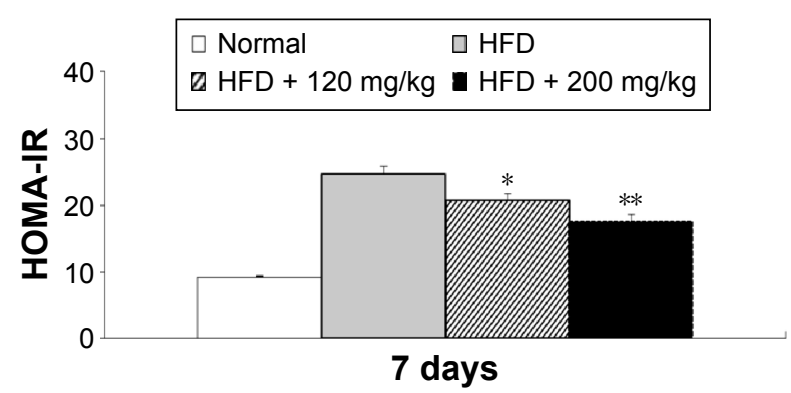

Figure 4 HOMA-IR analysis in HFD-fed diabetic rats after the 7-day experimental period.

Notes: The values represent the mean \pm SEM $(n=8) . * P<0.05$ and $* * p<0.0$ I compared to the HFD group, which received the same volume of vehicle.

Abbreviations: HOMA-IR, homeostatic model assessment for insulin resistance; HFD, high-fat diet; SEM, standard error of the mean.
Furthermore, in the HFD-fed diabetic rats, RA decreased the HOMA-IR index in a dose-related manner (Figure 4). Therefore, RA treatment for 7 days reversed insulin resistance in HFD-fed rats.

\section{Effects of RA on hepatic gluconeogenesis activity via PEPCK expression and on insulin sensitivity via GLUT4 expression}

The protein expression levels of PEPCK and GLUT4 were analyzed in STZ-induced diabetic rats and HFD-fed diabetic rats using Western blot analysis. PEPCK expression was markedly increased in isolated liver cells (Figures 5 and 6 ) in both the STZ-induced diabetic rats and the HFD-fed diabetic rats. RA treatment reduced hepatic PEPCK expression in both groups, suggesting that RA increased gluconeogenesis in the livers of diabetic rats. However, the decrease in PEPCK levels in the STZ group was more significant than the decrease in the HFD group. Additionally, we examined GLUT4 protein expression in muscle tissues to explore changes in glucose uptake (Figure 6), and we found that GLUT4 expression was significantly lower in both the STZ-induced diabetic rats and the HFD-fed diabetic rats. RA treatment markedly increased GLUT4 expression in both groups. Therefore, RA increased GLUT4 expression in skeletal muscle.

\section{Effect of RA on body weight, water intake, and food intake}

To investigate the long-term effects of RA in diabetic rats, changes in body weight and daily food and water intake were measured in both the STZ and the HFD groups, and the results are shown in Table 2. The administration of RA (120, 160, or $200 \mathrm{mg} / \mathrm{kg}$ ) for 28 days caused a significant decrease in body weight in the HFD group, but there was no significant difference in daily food or water intake. Furthermore, RA had no significant effect on body weight or daily food or water intake in the normal (control) and STZ groups.

\section{Discussion}

Diabetic disorders are metabolic diseases that are characterized by prolonged $\mathrm{HG}$, mainly as a result of the dysfunctional secretion and action of insulin. Glucose-stimulated insulin secretion occurs mainly in $\beta$-cells in the islets of Langerhans of the pancreas, and this process enhances glucose uptake. ${ }^{15}$ Therefore, the main diabetes treatment strategies are designed to maintain blood glucose levels, enhance $\beta$-cell function, and reduce insulin resistance to prevent complications. Meanwhile, medications used to treat diabetic disorders must not 


\section{A}
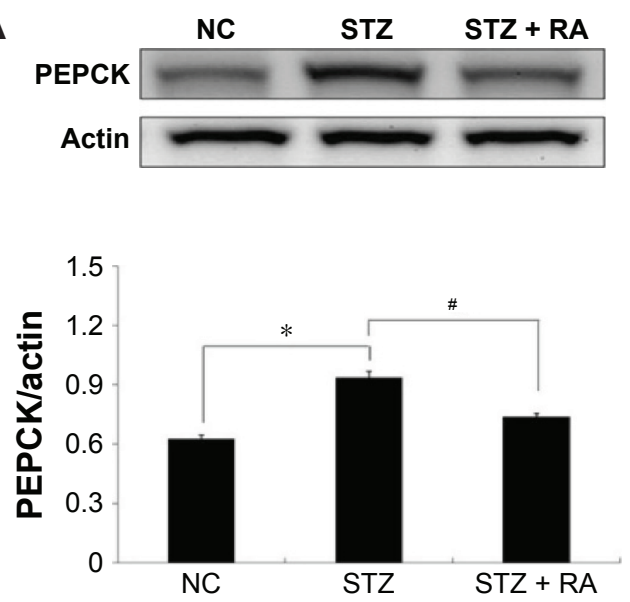

B
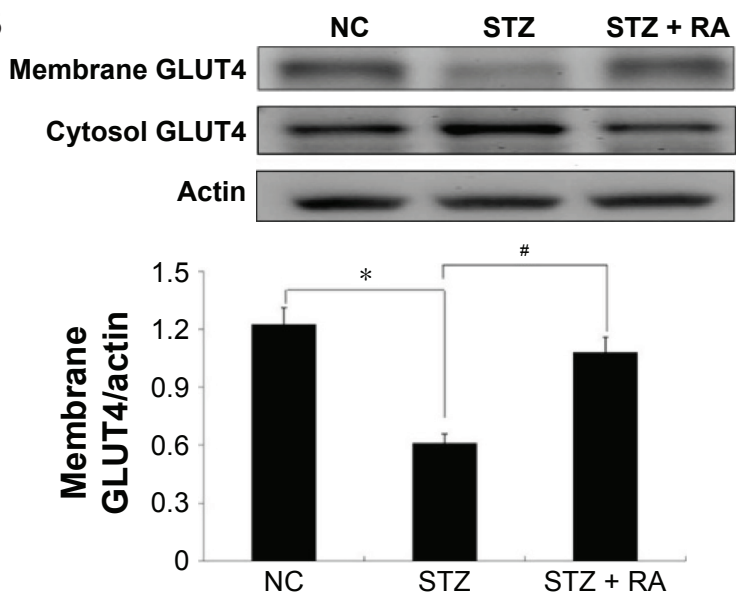

Figure 5 Effect of RA on the PEPCK protein levels in the liver and GLUT4 protein levels in the skeletal muscle of STZ-induced diabetic rats.

Notes: Sample extracts were prepared from the NC, STZ, and STZ + RA groups. STZ rats were treated with RA (200 mg/kg) via intraperitoneal injection for 7 days. (A) The PEPCK expression level in the liver. (B) Immunoblots of GLUT4 levels in the cytosol and membrane fractions of skeletal muscle samples. GLUT4 protein levels in the rats fed control chow were used as the control for the relevant quantifications. Each bar represents the mean $\pm S E M(n=6)$. $* P<0.05$ vs $N C$ and $\# P<0.05$ vs $S T Z$.

Abbreviations: RA, rosmarinic acid; STZ, streptozocin; NC, normal control; SEM, standard error of the mean.

A
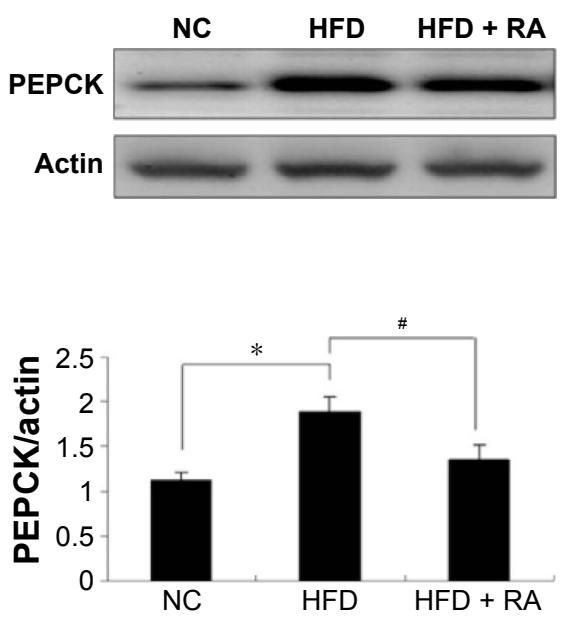

B
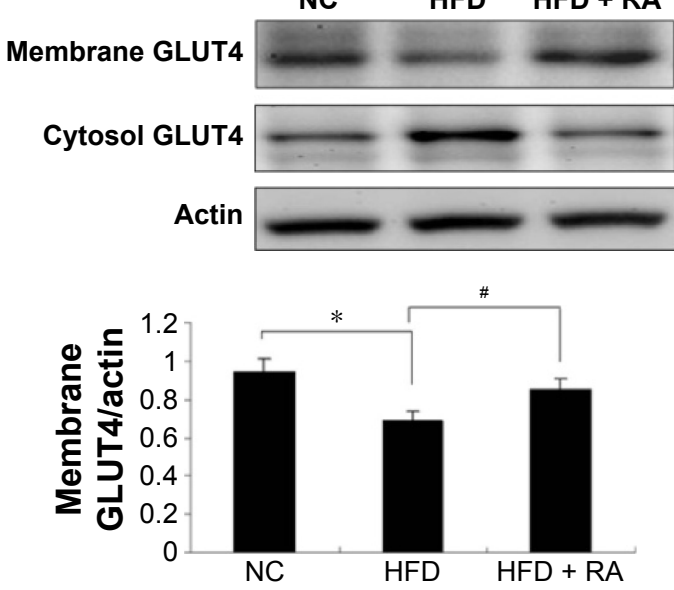

Figure 6 Effect of RA on PEPCK protein levels in the liver and GLUT4 protein levels in the skeletal muscle in HFD rats.

Notes: Sample extracts were prepared from the NC, HFD, and HFD + RA groups. HFD rats were treated with RA (200 mg/kg) via intraperitoneal injection for 7 days. (A) PEPCK expression levels in the liver. (B) Immunoblots showing GLUT4 levels in the cytosol and membrane fractions of skeletal muscle samples. The GLUT4 protein level in rats fed control chow was used as the control for the relevant quantifications. Each bar represents the mean $\pm S E M(n=6)$. $* P<0.05$ vs NC and ${ }^{*} P<0.05$ vs $H F D$. Abbreviations: RA, rosmarinic acid; HFD, high-fat diet; NC, normal control; SEM, standard error of the mean.

Table 2 Changes in body weight, food intake, and water intake in the experimental groups

\begin{tabular}{|c|c|c|c|c|c|c|}
\hline \multirow[t]{2}{*}{ Groups } & \multicolumn{2}{|c|}{ Body weight (g) } & \multicolumn{2}{|c|}{ Food intake (g) } & \multicolumn{2}{|c|}{ Water intake $(\mathrm{mL})$} \\
\hline & Day I & Day 28 & Day I & Day 28 & Day I & Day 28 \\
\hline Control & $194.10 \pm 4.27$ & $231.92 \pm 5.87$ & $21.29 \pm 1.22$ & $28.19 \pm 1.67$ & $30.18 \pm 4.05$ & $35.98 \pm 2.80$ \\
\hline RA $120 \mathrm{mg} / \mathrm{kg}$ & $193.28 \pm 4.39$ & $226.99 \pm 10.04$ & $21.59 \pm 2.11$ & $28.31 \pm 2.12$ & $30.57 \pm 3.37$ & $39.27 \pm 5.17$ \\
\hline RA $160 \mathrm{mg} / \mathrm{kg}$ & $192.78 \pm 4.68$ & $212.43 \pm 7.50$ & $22.8 I \pm 2.54$ & $29.88 \pm 2.33$ & $31.25 \pm 3.49$ & $36.27 \pm 3.78$ \\
\hline RA $200 \mathrm{mg} / \mathrm{kg}$ & $194.80 \pm 4.98$ & $215.52 \pm 8.13$ & $21.82 \pm 2.20$ & $28.39 \pm 2.59$ & $31.95 \pm 3.68$ & $39.22 \pm 5.31$ \\
\hline STZ & $202.81 \pm 3.43$ & $174.18 \pm 5.02$ & $36.02 \pm 2.70$ & $54.26 \pm 3.26$ & $149.20 \pm 6.45$ & $202.99 \pm 6.67$ \\
\hline $\mathrm{RA} 120 \mathrm{mg} / \mathrm{kg}$ & $201.45 \pm 4.47$ & $174.25 \pm 4.84$ & $36.0 I \pm 2.07$ & $52.67 \pm 3.58$ & $150.15 \pm 7.19$ & $207.25 \pm 6.23$ \\
\hline RA $160 \mathrm{mg} / \mathrm{kg}$ & $203.96 \pm 4.38$ & $176.67 \pm 5.79$ & $36.82 \pm 1.99$ & $52.05 \pm 3.05$ & $152.49 \pm 6.88$ & $208.9 I \pm 7.94$ \\
\hline RA $200 \mathrm{mg} / \mathrm{kg}$ & $202.12 \pm 4.54$ & $177.42 \pm 6.86$ & $36.84 \pm 2.26$ & $49.08 \pm 4.56$ & $155.33 \pm 8.99$ & $204.44 \pm 7.86$ \\
\hline HFD & $330.17 \pm 5.40$ & $389.82 \pm 8.95$ & $56.48 \pm 2.53$ & $77.99 \pm 3.28$ & $38.05 \pm 3.86$ & $42.85 \pm 3.71$ \\
\hline RA $120 \mathrm{mg} / \mathrm{kg}$ & $331.94 \pm 4.42$ & $365.26 \pm 6.65^{*}$ & $56.88 \pm 2.69$ & $74.90 \pm 3.66$ & $39.79 \pm 3.83$ & $42.05 \pm 5.71$ \\
\hline $\mathrm{RA} 160 \mathrm{mg} / \mathrm{kg}$ & $330.31 \pm 5.75$ & $359.34 \pm 9.09 *$ & $55.52 \pm 4.26$ & $73.02 \pm 3.43$ & $37 .|2 \pm 4.7|$ & $43.85 \pm 4.40$ \\
\hline RA $200 \mathrm{mg} / \mathrm{kg}$ & $334.00 \pm 8.21$ & $356.81 \pm 9.75^{*}$ & $58.00 \pm 2.63$ & $68.13 \pm 4.01$ & $39.79 \pm 3.83$ & $44.82 \pm 5.02$ \\
\hline
\end{tabular}

Notes: The values are shown as the mean $\pm \operatorname{SEM}(n=8) . * P<0.05$ compared with the untreated group (vehicle).

Abbreviations: RA, rosmarinic acid; STZ, streptozocin; HFD, high-fat diet; SEM, standard error of the mean. 
only lower blood glucose levels but also improve glucose homeostasis and insulin sensitivity. ${ }^{16}$

Chemically, RA (alpha-O-caffeoyl-3,4-dihydroxyphenyl lactic acid) is an ester of caffeic acid (CA) and 3,4-dihydroxyphenyl lactic acid. ${ }^{17}$ It is a common CA ester often found in plants that are the members of the Lamiaceae and Boraginaceae families. ${ }^{2}$ RA was originally isolated from rosemary ( $R$. officinalis) by ML Scarpatti and G Oriente in 1958. The chemical structure of RA is a dimer of CA and 3,4-dihydroxyphenyl lactic acid, with phenylpropanoid as the ester linkage. Biologically, it is synthesized from 4-coumaroyl-4'-hydroxyphenyllactate. In plants, RA constitutes an essential preformed defense compound against pathogens and herbivores. ${ }^{2,17}$ RA can be directly absorbed from the gastrointestinal tract. In rats, oral administration of RA produces seven metabolites in urine, including the intact form of RA and other metabolites, such as trans-CA 4- $O$-sulfate, trans-ferulic acid 4- $O$-sulfate, trans- $m$-coumaric acid 3- $O$-sulfate, trans-CA, trans- $m$-coumaric acid, and $m$-hydroxyphenylpropionic acid. ${ }^{18}$ These metabolites account for $-31.8 \%$ of total excretions in urine at 48 hours after oral administration of RA. Surprisingly, these metabolites are not found in bile, suggesting that RA is mainly excreted in urine. ${ }^{19}$

Antioxidant supplementation has been shown to improve the overall treatment outcome of diabetes, particularly chronic diabetes. Excessive oxidative stress worsens diabetic conditions and their complications. ${ }^{20}$ Persistent HG and elevated levels of free fatty acids contribute to oxidative stress and constitute a proximate cause of the onset and progression of diabetes and its complications. ${ }^{13}$ Therefore, in clinical practice, antioxidants are frequently included in complementary therapeutics. ${ }^{21}$

RA has been identified as a potent antioxidant. ${ }^{22,23}$ Extensive studies of RA have demonstrated its therapeutic properties, which mainly stem from its potent antioxidative effects. ${ }^{23}$ Those therapeutic properties include analgesic and anti-inflammatory effects, ${ }^{24}$ anticancer effects, ${ }^{25}$ and antidepressant, anxiolytic, and neuroprotective effects. ${ }^{26,27}$ RA can also disrupt the amyloid- $\beta$ conglomerates that are characteristic of Alzheimer's disease, ${ }^{28}$ and it has demonstrated antiviral ${ }^{29}$ as well as antibacterial and nematicidal activities. ${ }^{30}$ Additionally, no reports, including studies of hepatic and renal function and extensive blood cell counts, have identified any adverse effects of RA. ${ }^{31,32}$ Taken together, these data suggest that RA is a potential treatment for diabetes, particularly chronic diabetes.

In the present study, we focused on the efficacy of RA in treating diabetes. A previous paper showed that RA had little impact on glucose levels in normal rats. ${ }^{4}$ Therefore, experiments were conducted to explore the effects of RA in animal models of both type 1 and type 2 diabetes. Injecting STZ at a high dose $(>55 \mathrm{mg} / \mathrm{kg})$ can induce type 1-like diabetes $^{33}$ as a result of damage to pancreatic $\beta$-cells, which subsequently causes HG. Therefore, this procedure is frequently implemented to create animal models of type $1 \mathrm{DM} \cdot{ }^{34,35}$ Furthermore, HFD feeding produces insulin resistance ${ }^{36}$ and can be implemented to create animal models of type $2 \mathrm{DM} \cdot{ }^{33,34,37}$ In the present study, RA was shown to be effective at improving glucose homeostasis and insulin sensitivity in a dose-dependent manner.

The results from the PGTs, OGTTs, and ITTs showed that RA significantly improved glucose utilization and insulin sensitivity in normal, HFD-fed, and STZ-induced diabetic rats, all in a dose-dependent manner. The HOMA-IR provides an accurate measurement of insulin sensitivity in rats. ${ }^{38}$ The HOMA-IR evolved from the first HOMA, which was introduced in 1985, and included modifications to formulate a model of steady-state insulin and glucose domains by determining the physiological dose responses of insulin secretion and glucose uptake. In this assay, plasma glucose and insulin concentrations were used to estimate peripheral and hepatic glucose uptake and efflux. Thus, cell function defects could be estimated by altering the cell response to plasma glucose levels. ${ }^{39}$ In the present study, we adopted this model to estimate insulin resistance based on fasting glucose and insulin levels. The results of the HOMA-IR demonstrated that the RA-treated rats showed a significantly higher dose-dependent reduction than the rats in the control group, suggesting that RA has the potential to improve insulin resistance.

The liver is a primary organ that is involved in the regulation of blood glucose levels, and it does this by balancing glucose uptake and storage (glycogenesis) with glucose release (glycogenolysis and gluconeogenesis). Hepatic glucose production (HGP) occurs via the breakdown of glycogen and the de novo synthesis of glucose from noncarbohydrate precursors via gluconeogenesis. ${ }^{40-42}$ In contrast to acute $\mathrm{HG}$, in which HGP and gluconeogenic gene expression are suppressed, chronic HG is characterized by a progressive increase in basal HGP and is the main form of $\mathrm{HG}$ observed in diabetes. ${ }^{42}$ The rate of gluconeogenesis is modulated by enzymes, including PEPCK, fructose-1,6-biphosphatase, and glucose-6-phosphatase. ${ }^{40-42}$ However, the initial step in hepatic gluconeogenesis is catalyzed by PEPCK, ${ }^{43}$ which is upregulated during chronic $\mathrm{HG}$, including when this condition occurs in diabetic patients. Additionally, PEPCK is insulin-independent ${ }^{44}$ and can therefore be analyzed to investigate the amelioration of $\mathrm{HG}$ separately from increased insulin sensitivity. In the present study, the increase in the level of PEPCK expression in the liver was significantly 
lower after RA treatment. This effect of RA contributed to a reduction in $\mathrm{HG}$ in diabetic rats.

Skeletal muscle is a crucial site for glucose disposal activities. Glucose uptake is increased by insulin in skeletal muscles via the triggering of the translocation of GLUT4 from an intracellular deposit site to the membrane. Therefore, impaired GLUT4 translocation increases insulin resistance. ${ }^{45}$ Acute glucose-induced insulin resistance in rat skeletal muscle results in part from the consequential rapid decrease in GLUT4 in the plasma membrane. Glucose induces a decrease in plasma membrane levels of GLUT4 as a protective mechanism against excessive glucose uptake during $\mathrm{HG}$ accompanied by insulin resistance. ${ }^{46}$ Insulin sensitivity is enhanced by an increase in GLUT4 expression in skeletal muscle. ${ }^{47}$ In the present study, RA increased GLUT4 expression in skeletal muscle, and this process reversed the development of insulin resistance.

The limitations of the present study are mainly associated with the inclusion of data from only in vivo experiments. The cellular actions of RA were not analyzed in this report. Therefore, the mechanisms underlying the RA-induced increases of PEPCK and GLUT4 expression and the amelioration of HG, glucose levels, and insulin tolerance by upregulation of these proteins require additional studies for clarification. Additionally, many enzymes and pathways contribute to the regulation of HGP. Other key enzymes in the glucose phosphorylation/dephosphorylation, glycogen synthesis/degradation, and glycolysis and gluconeogenesis pathways must be analyzed in future studies. RA possesses antioxidant-like activity, and antioxidants are associated with decreased $\mathrm{HG}$ in animals. ${ }^{48,49}$ Therefore, the attenuation of HG by RA is likely to stem, in part, from its antioxidant-like activity. However, this hypothesis was not addressed in the present study.

\section{Conclusion}

The obtained data suggest that RA dose-dependently ameliorates $\mathrm{HG}$ and insulin resistance by decreasing PEPCK expression in the liver and increasing GLUT4 expression in muscles. For comparison, the clinical dose of RA in humans is $200 \mathrm{mg}$ / $\mathrm{kg},{ }^{50}$ and this level has been found to be conducive to most of its favorable biological properties, including anti-inflammatory and antiallergic effects. ${ }^{51}$ In the present study, a $200 \mathrm{mg} / \mathrm{kg}$ dose by body weight in rats was found to be the most effective dose for attaining RA's antidiabetic properties. This dose is similar to that used in previous studies of RA in rats ${ }^{52-54}$ but may not be appropriate for humans. Therefore, RA should be further investigated in clinical trials to evaluate its effectiveness as a new therapeutic agent for treating DM in humans.

\section{Acknowledgments}

The authors thank all the staff members of the Institute of Laboratory Animal Sciences at Kagoshima University (Frontier Science Research Center) for maintaining the animals in good condition. This study was supported by the Core Projects of the Diet and Obesity Translational Research of Kagoshima University Graduate School of Medical and Dental Sciences.

\section{Author contributions}

All authors contributed toward data analysis, drafting and critically revising the paper and agree to be accountable for all aspects of the work.

\section{Disclosure}

The authors report no conflicts of interest in this work.

\section{References}

1. Yeh GY, Eisenberg DM, Kaptchuk TJ, Phillips RS. Systematic review of herbs and dietary supplements for glycemic control in diabetes. Diabetes Care. 2003;26(4):1277-1294.

2. Petersen M. Rosmarinic acid: new aspects. Phytochem Rev. 2013;12(1): 207-227.

3. Azevedo MF, Lima CF, Fernandes-Ferreira M, Almeida MJ, Wilson JM, Pereira-Wilson C. Rosmarinic acid, major phenolic constituent of Greek sage herbal tea, modulates rat intestinal SGLT1 levels with effects on blood glucose. Mol Nutr Food Res. 2011;55(Supp1 1):S15-S25.

4. Jayanthy G, Subramanian S. Rosmarinic acid, a polyphenol, ameliorates hyperglycemia by regulating the key enzymes of carbohydrate metabolism in high fat diet - STZ induced experimental diabetes mellitus. Biomed Prev Nutr. 2014;4(3):431-437.

5. Sotnikova R, Okruhlicova L, Vlkovicova J, et al. Rosmarinic acid administration attenuates diabetes-induced vascular dysfunction of the rat aorta. J Pharm Pharmacol. 2013;65(5):713-723.

6. Farzadi L, Khaki A, Ghasemzadeh A, Ouladsahebmadarek E, Ghadamkheir E. Effect of rosmarinic acid on sexual behavior in diabetic male rats. Afr J Pharm Pharmacol. 2011;5(16):1906-1910.

7. Tavafi M, Ahmadvand H, Khalatbari A, Tamjidipoor A. Rosmarinic acid ameliorates diabetic nephropathy in uninephrectomized diabetic rats. Iran J Basic Med Sci. 2011;14(3):275-283.

8. Jiang WL, Xu Y, Zhang SP, Hou J, Zhu HB. Effect of rosmarinic acid on experimental diabetic nephropathy. Basic Clin Pharmacol Toxicol. 2012; 110(4):390-395.

9. Luan H, Kan Z, Xu Y, Lv C, Jiang W. Rosmarinic acid protects against experimental diabetes with cerebral ischemia: relation to inflammation response. J Neuroinflammation. 2013;10:28.

10. Hasanein P, Mohammad Zaheri L. Effects of rosmarinic acid on an experimental model of painful diabetic neuropathy in rats. Pharm Biol. 2014; 52(11):1398-1402.

11. Mushtaq N, Schmatz R, Pereira LB, et al. Rosmarinic acid prevents lipid peroxidation and increase in acetylcholinesterase activity in brain of streptozotocin-induced diabetic rats. Cell Biochem Funct. 2014;32(3): 287-293.

12. Govindaraj J, Sorimuthu Pillai S. Rosmarinic acid modulates the antioxidant status and protects pancreatic tissues from glucolipotoxicity mediated oxidative stress in high-fat diet: streptozotocin-induced diabetic rats. Mol Cell Biochem. 2015;404(1-2):143-159.

13. Matthews DR, Hosker JP, Rudenski AS, Naylor BA, Treacher DF, Turner RC. Homeostasis model assessment: insulin resistance and betacell function from fasting plasma glucose and insulin concentrations in man. Diabetologia. 1985;28(7):412-419. 
14. Runtuwene J, Amitani H, Amitani M, Asakawa A, Cheng KC, Inui A. Hydrogen-water enhances 5-fluorouracil-induced inhibition of colon cancer. Peer J. 2015;3:e859.

15. Beardsall K, Yuen K, Williams R, Dunger D. Applied physiology of glucose control. Curr Paediatr. 2006;16(6):434-438.

16. Wilcox G. Insulin and insulin resistance. Clin Biochem Rev. 2005;26(2): 19-39.

17. Petersen M, Simmonds MS. Rosmarinic acid. Phytochemistry. 2003; 62(2):121-125.

18. Lai XJ, Zhang L, Li JS, et al. Comparative pharmacokinetic and bioavailability studies of three salvianolic acids after the administration of Salviae miltiorrhizae alone or with synthetical borneol in rats. Fitoterapia. 2011;82(6):883-888.

19. Nakazawa T, Ohsawa K. Metabolism of rosmarinic acid in rats. J Nat Prod. 1998;61(8):993-996.

20. Giacco F, Brownlee M. Oxidative stress and diabetic complications. Circ Res. 2010;107(9):1058-1070.

21. Bradley R, Oberg EB, Calabrese C, Standish LJ. Algorithm for complementary and alternative medicine practice and research in type 2 diabetes. J Altern Complement Med. 2007;13(1):159-175.

22. Fadel O, El Kirat K, Morandat S. The natural antioxidant rosmarinic acid spontaneously penetrates membranes to inhibit lipid peroxidation in situ. Biochim Biophys Acta. 2011;1808(12):2973-2980.

23. Zhu F, Asada T, Sato A, Koi Y, Nishiwaki H, Tamura H. Rosmarinic acid extract for antioxidant, antiallergic, and alpha-glucosidase inhibitory activities, isolated by supramolecular technique and solvent extraction from perilla leaves. J Agric Food Chem. 2014;62(4):885-892.

24. Lucarini R, Bernardes WA, Ferreira DS, et al. In vivo analgesic and anti-inflammatory activities of Rosmarinus officinalis aqueous extracts, rosmarinic acid and its acetyl ester derivative. Pharm Biol. 2013;51(9): 1087-1090.

25. Karthikkumar V, Sivagami G, Vinothkumar R, Rajkumar D, Nalini N. Modulatory efficacy of rosmarinic acid on premalignant lesions and antioxidant status in 1,2-dimethylhydrazine induced rat colon carcinogenesis. Environ Toxicol Pharmacol. 2012;34(3):949-958.

26. Pereira P, Tysca D, Oliveira P, da Silva Brum LF, Picada JN, Ardenghi P. Neurobehavioral and genotoxic aspects of rosmarinic acid. Pharmacol Res. 2005;52(3):199-203.

27. Ticli FK, Hage LI, Cambraia RS, et al. Rosmarinic acid, a new snake venom phospholipase A2 inhibitor from Cordia verbenacea (Boraginaceae): antiserum action potentiation and molecular interaction. Toxicon. 2005;46(3):318-327.

28. Airoldi C, Sironi E, Dias C, et al. Natural compounds against Alzheimer's disease: molecular recognition of $A \beta 1-42$ peptide by Salvia sclareoides extract and its major component, rosmarinic acid, as investigated by NMR. Chem Asian J. 2013;8(3):596-602.

29. Swarup V, Ghosh J, Ghosh S, Saxena A, Basu A. Antiviral and antiinflammatory effects of rosmarinic acid in an experimental murine model of Japanese encephalitis. Antimicrob Agents Chemother. 2007;51(9):3367-3370.

30. Wang J, Pan X, Han Y, Guo D, Guo Q, Li R. Rosmarinic acid from eelgrass shows nematicidal and antibacterial activities against pine wood nematode and its carrying bacteria. Mar Drugs. 2012;10(12): 2729-2740.

31. Takano H, Osakabe N, Sanbongi C, et al. Extract of perilla frutescens enriched for rosmarinic acid, a polyphenolic phytochemical, inhibits seasonal allergic rhinoconjunctivitis in humans. Exp Biol Med (Maywood). 2004;229(3):247-254.

32. Hu G, Walls RS, Bass D, et al. The Chinese herbal formulation biminne in management of perennial allergic rhinitis: a randomized, doubleblind, placebo-controlled, 12-week clinical trial. Ann Allergy Asthma Immunol. 2002;88(5):478-487.

33. Srinivasan K, Viswanad B, Asrat L, Kaul CL, Ramarao P. Combination of high-fat diet-fed and low-dose streptozotocin-treated rat: a model for type 2 diabetes and pharmacological screening. Pharmacol Res. 2005; 52(4):313-320.
34. King AJ. The use of animal models in diabetes research. Br J Pharmacol. 2012;166(3):877-894.

35. Srinivasan K, Ramarao P. Animal models in type 2 diabetes research: an overview. Indian J Med Res. 2007;125(3):451-472.

36. Winzell MS, Ahrén B. The high-fat diet-fed mouse: a model for studying mechanisms and treatment of impaired glucose tolerance and type 2 diabetes. Diabetes. 2004;53(Suppl 3):S215-S219.

37. Cacho J, Sevillano J, de Castro J, Herrera E, Ramos MP. Validation of simple indexes to assess insulin sensitivity during pregnancy in Wistar and Sprague-Dawley rats. Am J Physiol Endocrinol Metab. 2008;295(5): E1269-E1276.

38. Wallace TM, Levy JC, Matthews DR. Use and abuse of HOMA modeling. Diabetes Care. 2004;27(6):1487-1495.

39. Nordlie RC, Foster JD, Lange AJ. Regulation of glucose production by the liver. Annu Rev Nutr. 1999;19:379-406.

40. Surwit RS, Kuhn CM, Cochrane C, McCubbin JA, Feinglos MN. Diet-induced type II diabetes in C57BL/6J mice. Diabetes. 1988;37(9): 1163-1167.

41. Vidal-Puig A, O'Rahilly S. Metabolism. Controlling the glucose factory. Nature. 2001;413(6852):125-126.

42. Shao J, Qiao L, Janssen RC, Pagliassotti M, Friedman JE. Chronic hyperglycemia enhances PEPCK gene expression and hepatocellular glucose production via elevated liver activating protein/liver inhibitory protein ratio. Diabetes. 2005;54(4):976-984.

43. Quinn PG, Yeagley D. Insulin regulation of PEPCK gene expression: a model for rapid and reversible modulation. Curr Drug Targets Immune Endocr Metabol Disord. 2005;5(4):423-437.

44. Scott DK, O'Doherty RM, Stafford JM, Newgard CB, Granner DK. The repression of hormone-activated PEPCK gene expression by glucose is insulin-independent but requires glucose metabolism. J Biol Chem. 1998;273(37):24145-24151.

45. Tremblay F, Lavigne C, Jacques H, Marette A. Defective insulininduced GLUT4 translocation in skeletal muscle of high fat-fed rats is associated with alterations in both Akt/protein kinase B and atypical protein kinase C (zeta/lambda) activities. Diabetes. 2001;50(8):1901-1910.

46. Marette A, Dimitrakoudis D, Shi Q, Rodgers CD, Klip A, Vranic M. Glucose rapidly decreases plasma membrane GLUT4 content in rat skeletal muscle. Endocrine. 1999;10(1):13-18.

47. Richter EA, Hargreaves M. Exercise, GLUT4, and skeletal muscle glucose uptake. Physiol Rev. 2013;93(3):993-1017.

48. Gulati V, Harding IH, Palombo EA. Enzyme inhibitory and antioxidant activities of traditional medicinal plants: potential application in the management of hyperglycemia. BMC Complement Altern Med. 2012; 12:77.

49. Shori AB. Screening of antidiabetic and antioxidant activities of medicinal plants. J Integr Med. 2015;13(5):297-305.

50. Baba S, Osakabe N, Natsume M, et al. Absorption, metabolism, degradation and urinary excretion of rosmarinic acid after intake of perilla frutescens extract in humans. Eur J Nutr. 2005;44(1):1-9.

51. Osakabe N, Takano H, Sanbongi C, et al. Anti-inflammatory and anti-allergic effect of rosmarinic acid (RA); inhibition of seasonal allergic rhinoconjunctivitis (SAR) and its mechanism. Biofactors. 2004; 21(1-4):127-131.

52. Osakabe N, Yasuda A, Natsume M, et al. Rosmarinic acid, a major polyphenolic component of perilla frutescens, reduces lipopolysaccharide (LPS)-induced liver injury in d-galactosamine (d-GalN)-sensitized mice. Free Radic Biol Med. 2002;33(6):798-806.

53. Zou ZW, Xu LN, Tian JY. [Antithrombotic and antiplatelet effects of rosmarinic acid, a water-soluble component isolated from radix Salviae miltiorrhizae (danshen)]. Үао Хие Хие Вао. 1993;28(4):241-245. Chinese.

54. Makino T, Ono T, Liu N, Nakamura T, Muso E, Honda G. Suppressive effects of rosmarinic acid on mesangioproliferative glomerulonephritis in rats. Nephron. 2002;92(4):898-904. 


\section{Publish your work in this journal}

Drug Design, Development and Therapy is an international, peerreviewed open-access journal that spans the spectrum of drug design and development through to clinical applications. Clinical outcomes, patient safety, and programs for the development and effective, safe, and sustained use of medicines are a feature of the journal, which

has also been accepted for indexing on PubMed Central. The manuscript management system is completely online and includes a very quick and fair peer-review system, which is all easy to use. Visit http://www.dovepress.com/testimonials.php to read real quotes from published authors.

Submit your manuscript here: http://www.dovepress.com/drug-design-development-and-therapy-journal 